\title{
Discussion of "Predicting water permeability in sedimentary rocks from capillary imbibition and pore structure" by D. Benavente et al., Engineering Geology (2015) [doi: 10.1016/j.enggeo.2015.06.003]
}

Christopher Hall ${ }^{1, *}$, Andrea Hamilton ${ }^{2}$

Keywords: Permeability; Sorptivity; Imbibition; Limestones; Wettability

1

2

\footnotetext{
${ }^{*}$ Corresponding author

${ }^{1}$ School of Engineering, University of Edinburgh, United Kingdom

${ }^{2}$ Department of Civil and Environmental Engineering, University of Strathclyde, United Kingdom
} 


\section{Scaling relations}

The sorptivity depends on both the permeability and the capillary suction of the medium. Its composite character is shown, for example, in the GreenAmpt expression for the sorptivity, $S=(2 \phi K|\Psi|)^{1 / 2}$, where $\phi$ is the volumefraction porosity, $K$ the permeability of the wetted zone, and $\Psi$ the wet-front capillary pressure potential [2]. We may write this as

$$
S_{\star}=S /\left(\gamma_{\mathrm{L}} / \eta_{\mathrm{L}}\right)^{1 / 2}=\left(2 \phi k p_{\star}\right)^{1 / 2}
$$

where $S_{\star}$ is the intrinsic sorptivity, $\gamma_{\mathrm{L}}$ and $\eta_{\mathrm{L}}$ the surface tension and viscosity of the imbibed liquid, and $k=K \eta_{\mathrm{L}} /\left(\rho_{\mathrm{L}} g\right)$ the intrinsic permeability of the wetted zone. We define the reduced wet-front capillary pressure $p_{\star}=|\Psi| \rho_{\mathrm{L}} g / \gamma_{\mathrm{L}}$, with $\rho_{\mathrm{L}}$ the density of the imbibed liquid. The dimensions of the main quantities are: $S,\left[\mathrm{LT}^{-1 / 2}\right] ; S_{\star},\left[\mathrm{L}^{1 / 2}\right] ; k,\left[\mathrm{~L}^{2}\right] ;$ and $p_{\star},\left[\mathrm{L}^{-1}\right]$.

Although $S_{\star}$ depends on both $k$ and $p_{\star}$, the two latter quantities are highly correlated, since both are determined by the same underlying pore structure.

The permeability $k$ is proportional to the porosity $\phi$, and varies as the square of some characteristic length of the pore system, say $\lambda$ : that is, $k \sim \phi \lambda^{2}$. On the other hand, the capillary pressure $p_{\star} \sim 1 / \lambda$, and is independent of $\phi$. It follows then that $S_{\star} \sim \phi \lambda^{1 / 2}$. These relations are well known, and can be traced back at least as far as [6] (see also [4, 2]). These scalings also imply the further relation $k \sim S_{\star}^{4} \phi^{-3}$. 


\section{Empirical correlations}

In [1], the authors test several empirical correlations between $k, C$, $\phi$, and various measures of pore size such as a mean pore radius $r_{\mathrm{M}}$ obtained by mercury intrusion porosimetry. Among the more successful of these, they

find a best-fit regression equation $k=A C^{a} \phi^{b}$ where $A$ is a constant, $a=4.6$, $b=-2.1$, with $\phi$ the open (connected) porosity, and for which $R^{2}=0.93$. The sample size is small $(n=13)$, so that the standard uncertainties of the regression parameters are sizeable: $a=4.6 \pm 0.5$, and $b=-2.1 \pm 1.1$ (these uncertainties are calculated from our own regression analysis on the logarithmic form of the data in [1]). Since $S_{\star} \sim C$, this empirical regression equation is broadly consistent with the simple scaling $k \sim S_{\star}^{4} \phi^{-3}$ we derived earlier.

\section{Predictive models}

While simple scalings impose constraints on the functional form of relations between $k, S_{\star}, \phi$, and other variables, they do not yield predictive models. For that, fully explicit relations are required, notably to identify the lengthscale $\lambda$ with a measurable property. In [1], the authors derive a predictive equation (their Eqn 10) to estimate $k$. They take the Lucas-Washburn equation as their starting point, but their predictive equation does not depend on a tube-bundle permeability. The only structural assumptions are that the lengthscale $\lambda=r_{\mathrm{T}}$, the threshold pore radius, and that the reduced capillary pressure $p_{\star}=2 \cos (\theta) / \lambda$, where $\theta$ is a notional contact angle, and hence $\cos (\theta)$ is a wetting index. Neglecting partial-wetting effects for the moment, 
we can set $C=S_{\star} \rho_{\mathrm{L}}\left(\gamma_{\mathrm{L}} / \eta_{\mathrm{L}}\right)^{1 / 2}$ to see that the predictive equation of [1] is the same as Eqn 1 above.

\section{Caveats}

1. The scalings that we have mentioned apply strictly only to groups of materials with geometrically similar microstructures. They are the basis of the definition of Miller-similar or scale-heterogeneous materials in soil physics $[3,5]$. Given that groups of real materials such as carbonate rocks are not strictly similar in that sense, deviations from the predictive equation must be expected. Nonetheless, the agreement between experimental and predicted permeability in [1] is impressive, and it will be of great interest to see the results of further tests.

2. In using the scaling $k \sim S_{\star}^{4} \phi^{-3}$ to predict the conventional saturated permeability we assume implicitly that the state of saturation in capillary imbibition is the same as that in a test to measure the saturated permeability. This is not generally true, since the mean liquid content of the wetted zone in imbibition is below saturation as a result of air-trapping. However, if the permeabilities of the two states are in constant ratio the scaling holds.

3. In [1], the strong influence of wettability is rightly emphasized. Many carbonate rocks show evidence of partial (incomplete) wetting in imbibition tests with water (see for example [2]). Wetting indices vary considerably from stone to stone, and no doubt depend as much on the sample history as on the mineralogy. We suggest that for the purposes 
of estimating the permeability $k$ from $S_{\star}$, the effects of partial wetting can be eliminated if $S$ (and hence $S_{\star}$ ) is obtained not with water but with a low surface-tension liquid such as $n$-decane, where complete wetting is usually found.

\section{References}

[1] Benavente D., Pla C., Cueto N., Galvañ S., Martínez-Martínez J., García-del-Cura M.A., and Ordoñez S., Predicting water permeability in sedimentary rocks from capillary imbibition and pore structure, Eng. Geol. 2015, http://dx.doi.org/10.1016/j.enggeo.2015.06.003, (on-line).

[2] Hall C. and Hoff W.D., Water Transport in Brick, Stone and Concrete, second ed, 2012, Taylor \& Francis: London.

[3] Miller E.E and Miller R.D., Physical theory for capillary flow phenomena, J. Appl. Phys., 27, 1956, 324-332.

[4] Philip J.R., Field heterogeneity: some basic issues, Water Resour. Res., 16, 1980, 443-448.

[5] Philip J.R., Sorption and infiltration in heterogeneous media, Aust. J. Soil Res., 5, 1967, 1-10.

[6] D. Swartzendruber D., de Boodt M.F. and Kirkham D., Capillary intake of water and soil structure. Soil Sci. Soc. Am. Proc., 18, 1954, 1-7. 